\title{
The evolution of phase states of Universe to the present inert phase
}

\author{
Ilya F. Ginzburg ${ }^{a *}$, K.A. Kanishev ${ }^{a}$, M. Krawczyk ${ }^{b}$ and D. Sokołowska ${ }^{b \dagger}$ \\ ${ }^{a}$ Sobolev Institute of Mathematics and Novosibirsk State University, Novosibirsk, Russia \\ ${ }^{b}$ Institute of Theoretical Physics, University of Warsaw, Warsaw, Poland \\ E-mail: ginzburg.math.nsc.ru, kkanishev@gmail.com, \\ Maria.Krawczykdfuw.edu.pl, dsokdfuw.edu.pl
}

\begin{abstract}
We assume that current state of the Universe is described by the Inert Doublet Model, containing two scalar doublets, one of which is responsible for EWSB and masses of particles and the second one having no couplings to fermions and being responsible for dark matter. We consider possible evolutions of the Universe to this state during cooling down of the Universe after inflation. We found that in the past Universe could pass through phase states having no DM candidate. In the evolution via such states in addition to a possible EWSB phase transition (2-nd order) the Universe sustained one 1-st order phase transition or two phase transitions of the 2-nd order.
\end{abstract}

The XIXth International Workshop on High Energy Physics and Quantum Field Theory 8-15 September 2010

Golitsyno, Moscow, Russia

\footnotetext{
${ }^{*}$ Speaker.

$\dagger$ We are thankful to I. Ivanov, M. Dubinin and R. Nevzorov for useful discussions. Work was partly supported by Polish Ministry of Science and Higher Education Grant N N202 230337. The work of MK and DS was supported in addition by EU Marie Curie Research Training Network HEPTOOLS, under contract MRTN-CT-2006-035505, FLAVIAnet contract No. MRTN-CT-2006-035482. The work of IG and KK was also supported by grants RFBR 08-02-00334a, 10-02-8579, NSh-3810.2010.2 and Program of Dept. of Phys. Sc. RAS "Experimental and theoretical studies of fundamental interactions related to LHC."
} 


\section{Introduction}

One of the widely discussed models for Dark Matter (DM) particles is the Inert Doublet Model (IDM) [四]. The model contains "standard" scalar (Higgs) doublet $\phi_{S}$, responsible for electroweak symmetry breaking and masses of fermions and gauge bosons as in the Standard Model (SM), and scalar doublet, $\phi_{D}$, which doesn't receive vacuum expectation value (v.e.v.) and doesn't couple to fermions. (Our notations are similar to those in the $2 H D M$ with $\phi_{1} \rightarrow \phi_{S}, \phi_{2} \rightarrow \phi_{D}$.) More complete version of this report is given in [2]]. Here four degrees of freedom of the Higgs doublet $\phi_{S}$ are as in the SM: three Goldstone modes become longitudinal components of the EW gauge bosons and one mode becomes the Higgs boson $h_{S}$. All the components of the scalar doublet $\phi_{D}$ are realized as massive scalar $D$-particles: two charged $D^{ \pm}$and two neutral ones $D_{H}$ and $D_{A}$. By construction, they possess a conserved multiplicative quantum number and therefore the lightest particle among them can be considered as a candidate for DM particle. Assuming that DM particles are neutral, we have

$$
M_{D^{ \pm}}, M_{D_{A}} \geq M_{D_{H}} \text { or } M_{D^{ \pm}}, M_{D_{H}} \geq M_{D_{A}} .
$$

Possible masses of $D$-particles are constrained by the accelerator and astrophysical data (see e.g. [3] ).

Assuming that the current state of the Universe is described by IDM, we discuss possible variants of the history of the phase states of Universe during its cooling down after inflation. In some respects, this analysis can be considered as particular case of analysis [四], [曹]. We use below some results and notations from [䧃]-[焑].

\section{The Lagrangian}

The electroweak symmetry breaking via the Higgs mechanism is described by the Lagrangian

$$
\mathscr{L}=\mathscr{L}_{g f}^{S M}+T-V+\mathscr{L}_{Y}\left(\psi_{f}, \phi_{S}\right)
$$

Here, $\mathscr{L}_{g f}^{S M}$ describes the $S U(2) \times U(1)$ Standard Model interaction of gauge bosons and fermions, which is independent on the realization of the Higgs mechanism, $T$ is the standard kinetic term for two scalar doublets $\phi_{S}$ and $\phi_{D}$ and the potential $V$ with these two scalars. The $\mathscr{L}_{Y}$ describes the Yukawa interaction of fermions $\psi_{f}$ with only one scalar doublet $\phi_{S}$ in the same form as in the SM.

Potential. The potential must be $Z_{2}$ symmetric in order to describe IDM. Without loss of generality it can be written in the form with all real parameters ${ }^{1}$

$$
\begin{gathered}
V=-\frac{1}{2}\left[m_{11}^{2}\left(\phi_{S}^{\dagger} \phi_{S}\right)+m_{22}^{2}\left(\phi_{D}^{\dagger} \phi_{D}\right)\right]+\frac{\lambda_{1}}{2}\left(\phi_{S}^{\dagger} \phi_{S}\right)^{2}+\frac{\lambda_{2}}{2}\left(\phi_{D}^{\dagger} \phi_{D}\right)^{2}+\lambda_{3}\left(\phi_{S}^{\dagger} \phi_{S}\right)\left(\phi_{D}^{\dagger} \phi_{D}\right)+ \\
+\lambda_{4}\left(\phi_{S}^{\dagger} \phi_{D}\right)\left(\phi_{D}^{\dagger} \phi_{S}\right)+\frac{\lambda_{5}}{2}\left[\left(\phi_{S}^{\dagger} \phi_{D}\right)^{2}+\left(\phi_{D}^{\dagger} \phi_{S}\right)^{2}\right], \quad \lambda_{5}<0 .
\end{gathered}
$$

The IDM is realized in some regions of parameters of this potential. To study thermal evolution, we will consider also other possible vacuum states of such potential, at another values of parameters.

\footnotetext{
${ }^{1}$ In the general $Z_{2}$ symmetric potential the last term has a form $\left[\tilde{\lambda}_{5}\left(\phi_{S}^{\dagger} \phi_{D}\right)^{2}+\tilde{\lambda}_{5}^{*}\left(\phi_{D}^{\dagger} \phi_{S}\right)^{2}\right]$. The physical content of theory cannot be changed by the global phase rotation $\phi_{a} \rightarrow \phi_{a} e^{i \alpha_{a}}(a=S, D)$. Starting with an arbitrary complex $\tilde{\lambda}_{5}=\left|\tilde{\lambda}_{5}\right| e^{i \rho}$ we select $\alpha_{S}-\alpha_{D}=\rho / 2+\pi / 2$, to get (․2.) with negative $\lambda_{5}=-\left|\tilde{\lambda}_{5}\right|$.
} 
To make some equations shorter, we use the abbreviations:

$$
\lambda_{345}=\lambda_{3}+\lambda_{4}+\lambda_{5}, \quad R=\frac{\lambda_{345}}{\sqrt{\lambda_{1} \lambda_{2}}} .
$$

Discrete symmetries. This potential (‥2) is invariant under two discrete symmetry transformations of a $Z_{2}$ type, called respectively $S$-transformation and $D$-transformation (here $\mathrm{SM}$ denote the SM fermions and gauge bosons):

$$
\begin{array}{cc}
S: \phi_{S} \stackrel{S}{\rightarrow}-\phi_{S}, \quad \phi_{D} \stackrel{S}{\rightarrow} \phi_{D}, \quad S M \stackrel{S}{\rightarrow} S M, \\
D: \phi_{S} \stackrel{D}{\rightarrow} \phi_{S}, \quad \phi_{D} \stackrel{D}{\rightarrow}-\phi_{D}, \quad S M \stackrel{D}{\rightarrow} S M .
\end{array}
$$

For the EW symmetric phase (with $\left\langle\phi_{S}\right\rangle=\left\langle\phi_{D}\right\rangle=0$ this invariance results in the D-parity and $S$-parity conservation in the processes involving only scalars and gauge bosons. The Yukawa term violates $S$-symmetry, while it respects $D$-symmetry in any order of perturbation theory.

Positivity constraints. To have a stable vacuum, the potential must be positive at large quasiclassical values of fields $\left|\phi_{i}\right|$ (positivity constraints), for an arbitrary direction in the $\left(\phi_{S}, \phi_{D}\right)$ plane. These conditions limit possible values of $\lambda_{i}$ (see e.g. [ [0] ). In terms of parameters ([2.3]) positivity constraints which are needed in our analysis, can be written as

$$
\lambda_{1}>0, \quad \lambda_{2}>0, \quad R+1>0
$$

\section{Thermal evolution}

Potential. Since the Hubble constant is small, we assume a statistical equilibrium at every temperature $T$. In this approximation, at the finite temperature, the ground state of system is given by a minimum of the Gibbs potential

$$
V_{G}=\operatorname{Tr}\left(V e^{-\hat{H} / T}\right) / \operatorname{Tr}\left(e^{-\hat{H} / T}\right) .
$$

In the first nontrivial approximation and high enough temperature the obtained Gibbs potential has the same form as the basic potential $V(\mathbb{2} 2)$, i. e. as the potential at zero temperature. The coefficients $\lambda^{\prime} s$ of the quartic terms in the potential $V_{G}$ and $V$ coincide, while the mass terms vary with temperature $T$, as follows

$$
\begin{gathered}
m_{11}^{2}(T)=m_{11}^{2}-c_{1} T^{2}, \quad m_{22}^{2}(T)=m_{22}^{2}-c_{2} T^{2}, \\
c_{1}=\frac{3 \lambda_{1}+2 \lambda_{3}+\lambda_{4}}{12}+\frac{3 g^{2}+g^{\prime 2}}{32}+\frac{g_{t}^{2}+g_{b}^{2}}{8}, \quad c_{2}=\frac{3 \lambda_{2}+2 \lambda_{3}+\lambda_{4}}{12}+\frac{3 g^{2}+g^{\prime 2}}{32} .
\end{gathered}
$$

Here $g$ and $g^{\prime}$ are the EW gauge couplings, $g_{t} \approx 1$ and $g_{b} \approx 0.03$ are values of the SM Yukawa couplings for $t$ and $b$ quarks, respectively.

Generally each of coefficients $c_{1}$ and $c_{2}$ can be either positive or negative. However, in virtue of positivity conditions (2., their sum is positive (even neglecting positive contributions from gauge bosons $\mathrm{W} / \mathrm{Z}$ and fermions),

$$
c_{2}+c_{1}>0
$$


We will show later on that for a realization of the present inert vacuum with neutral dark matter particle one needs $\lambda_{4}+\lambda_{5}<0$ (5.4). Therefore, at $R>0$ we have $\lambda_{3}>0$. Taking into account that $\lambda_{5}<0$ ([2.2) , we obtain that $c_{1}>0, c_{2}>0$. At $R<0$ there are no constraints on signs of $c_{1,2}$ :

$$
\begin{gathered}
R>0: \quad c_{1}>0, \quad c_{2}>0 ; \\
R<0: \quad \text { arbitrary signs of } c_{1,2} .
\end{gathered}
$$

Yukawa interaction. The form of Yukawa interaction and values of Yukawa couplings don't vary during thermal evolution.

\section{Extrema of the potential}

Following [四] we first consider extrema of the potential ([2.2]) at arbitrary values of parameters. The extrema conditions:

$$
\partial V /\left.\partial \phi_{i}\right|_{\phi_{i}=\left\langle\phi_{i}\right\rangle}=0, \partial V /\left.\partial \phi_{i}^{\dagger}\right|_{\phi_{i}=\left\langle\phi_{i}\right\rangle}=0 \quad(i=S, D)
$$

define the extremum values $\left\langle\phi_{S}\right\rangle$ and $\left\langle\phi_{D}\right\rangle$ of the fields $\phi_{S}$ and $\phi_{D}$, respectively. The extremum with the lowest energy (the global minimum of the potential) realizes the vacuum state of the system. Other extrema are saddle points, maxima or local minima of the potential.

For each electroweak symmetry violating extremum with $\left\langle\phi_{S}\right\rangle \neq 0$, one can choose the $z$ axis in the weak isospin space so that $\left\langle\phi_{S}\right\rangle \sim\left(\begin{array}{c}0 \\ v_{S}\end{array}\right)$, with real, nonnegative $v_{S}$ (choosing a "neutral direction" in the weak isospin space). Therefore, the most general solution of (4) can be written as

$$
\left\langle\phi_{S}\right\rangle=\frac{1}{\sqrt{2}}\left(\begin{array}{c}
0 \\
v_{S}
\end{array}\right), \quad\left\langle\phi_{D}\right\rangle=\frac{1}{\sqrt{2}}\left(\begin{array}{c}
u \\
v_{D}
\end{array}\right) .
$$

Neutral extrema. The solutions of (4. (1) with $u=0$ are called neutral extrema, as they respect $U$ (1) symmetry of electromagnetism. For these extrema the conditions (4.]) can be written as a system of two degenerate cubic equations with four solutions:

$$
v_{S}\left(-m_{11}^{2}+\lambda_{1} v_{S}^{2}+\lambda_{345} v_{D}^{2}\right)=0, \quad v_{D}\left(-m_{22}^{2}+\lambda_{2} v_{D}^{2}+\lambda_{345} v_{S}^{2}\right)=0, \quad v_{S}^{2}, v_{D}^{2} \geqslant 0 .
$$

This system has four solutions (here $\mathscr{E}_{a}$ are extrema energies):

$$
\begin{aligned}
& \text { EWs : EW symmetric } v_{D}=0, \quad v_{S}=0, \quad \mathscr{E}_{E W s}=0 ; \\
& \boldsymbol{I}_{1}: \quad \text { inert } \quad v_{D}=0, v^{2} \equiv v_{S}^{2}=\frac{m_{11}^{2}}{\lambda_{1}}, \quad \mathscr{E}_{I_{1}}=-\frac{m_{11}^{4}}{8 \lambda_{1}} ; \\
& \boldsymbol{I}_{2}: \quad \text { inert }- \text { like } \quad v_{S}=0, v^{2} \equiv v_{D}^{2}=\frac{m_{22}^{2}}{\lambda_{2}}, \quad \mathscr{E}_{I_{2}}=-\frac{m_{22}^{4}}{8 \lambda_{2}} ; \\
& \boldsymbol{M}: \quad \text { mixed }\left\{\begin{array}{c}
v_{S}^{2}=\frac{m_{11}^{2} \lambda_{2}-\lambda_{345} m_{22}^{2}}{\lambda_{1} \lambda_{2}-\lambda_{345}^{2}}, \quad v_{D}^{2}=\frac{m_{22}^{2} \lambda_{1}-\lambda_{345} m_{11}^{2}}{\lambda_{1} \lambda_{2}-\lambda_{345}^{2}}, \\
\mathscr{E}_{M}=\frac{-m_{11}^{4} \lambda_{2}+2 \lambda_{345} m_{11}^{2} m_{22}^{2}-m_{22}^{4} \lambda_{1}}{8\left(\lambda_{1} \lambda_{2}-\lambda_{345}^{2}\right)} .
\end{array}\right.
\end{aligned}
$$


If one of these equations gives $v_{S}^{2}<0$ or $v_{D}^{2}<0$, the corresponding extremum is absent.

The energy differences between $I_{1,2}$ and $M$ extrema are

$$
\mathscr{E}_{I_{1}}-\mathscr{E}_{M}=\frac{\left(m_{11}^{2} \lambda_{345}-m_{22}^{2} \lambda_{1}\right)^{2}}{8 \lambda_{1}^{2} \lambda_{2}\left(1-R^{2}\right)} ; \quad \mathscr{E}_{I_{2}}-\mathscr{E}_{M}=\frac{\left(m_{22}^{2} \lambda_{345}-m_{11}^{2} \lambda_{2}\right)^{2}}{8 \lambda_{1} \lambda_{2}^{2}\left(1-R^{2}\right)}
$$

Charge breaking extremum. For $u \neq 0$ the extremum violates not only EW symmetry but also the $U(1)$ electromagnetic symmetry, leading to the electric charge non-conservation. This extremum can realize vacuum state only if $\lambda_{4}+\lambda_{5}>0$ [四, 困]. We will see later on that at this condition the DM particle can not be neutral, that contradicts (ㅁ. $(\mathbb{L})$.

\section{Vacuum states}

Below we describe briefly the properties of neutral extrema, listed above, provided in each case that it realizes a true vacuum.

\subsection{Electroweak symmetric vacuum $E W s,\left\langle\phi_{S}\right\rangle=\left\langle\phi_{D}\right\rangle=0$}

The electroweak symmetric extremum exists for all values of parameters of the potential (2.2). This extremum is a minimum, realizing vacuum state, at

$$
m_{11}^{2}<0, \quad m_{22}^{2}<0 .
$$

In this case, gauge bosons and fermions are massless, while scalar doublets $\phi_{S}$ and $\phi_{D}$ have masses equal to $\left|m_{11}\right| / \sqrt{2}$ and $\left|m_{22}\right| / \sqrt{2}$, respectively.

\subsection{Inert vacuum $I_{1},\left\langle\phi_{D}\right\rangle=0$}

In the case when $I_{1}$ extremum realizes vacuum, the Inert Doublet Model describes reality. The standard field decomposition near $I_{1}$ extremum has a form

$$
\phi_{S}=\left(\begin{array}{c}
G^{+} \\
\frac{v+h_{S}+i G}{\sqrt{2}}
\end{array}\right), \quad \phi_{D}=\left(\begin{array}{c}
D^{+} \\
\frac{D_{H}+i D_{A}}{\sqrt{2}}
\end{array}\right)
$$

where $G^{ \pm}$and $G$ are Goldstone modes, while $h_{S}$ and $D=D_{H}, D_{A}, D^{ \pm}$are scalar particles. Here the Higgs particle $h_{S}$ interacts with the fermions and gauge bosons just as the Higgs boson in the SM. $D$-particles don't interact with fermions. Neither there are interactions of $D$-particles with gauge bosons $V$ of the type $D_{i} V_{1} V_{2}$.

Symmetry properties. This vacuum is invariant under the $D$-transformation ([2.5). Therefore the $D$-parity is conserved. Hence, the lightest $D$-particle is stable, being a good DM candidate. (In this state the $S$-symmetry (2.4) is broken.)

Allowed region of parameters. The inert extremum exists if only $m_{11}^{2}>0$ (4.5). In accordance with (4.5) and (4.6), the extremum $I_{1}$ can be a vacuum only if $m_{11}^{2} / \sqrt{\lambda_{1}}>m_{22}^{2} / \sqrt{\lambda_{2}}$. Additional condition arises from a comparison of $I_{1}$ and $M$ extrema. In virtue of (4.8) at $R^{2}>1$ the extremum $M$ can exist but its energy is larger than energy of $I_{1}$ extremum - so that the extremum $I_{1}$ realizes vacuum. At $R^{2}<1$ the inert extremum still can be a vacuum, in the case when the mixed extremum 
does not exist, i. e. if at least one of quantities $v_{S}^{2}, v_{D}^{2}$ defined by eq. (4.]) is negative. Note, that due to the positivity constraint $1+R>0$ (R.G) in the case when $R^{2}>1$ we have $R>1$. For the opposite case, with $R^{2}<1$, the quantity $R$ can be either positive or negative.

Particle properties. The quadratic part of the potential written in terms of physical fields $h_{S}, D_{H}, D_{A}$ and $D^{ \pm}(5.2)$ gives the masses of scalars:

$$
\begin{gathered}
M_{h_{S}}^{2}=\lambda_{1} v^{2}=m_{11}^{2}, \quad M_{D^{ \pm}}^{2}=\frac{\lambda_{3} v^{2}-m_{22}^{2}}{2}, \\
M_{D_{A}}^{2}=M_{D^{ \pm}}^{2}+\frac{\lambda_{4}-\lambda_{5}}{2} v^{2}, \quad M_{D_{H}}^{2}=M_{D^{ \pm}}^{2}+\frac{\lambda_{4}+\lambda_{5}}{2} v^{2} .
\end{gathered}
$$

The requirement that lightest $D$-particle is a neutral one $(\mathbb{L}$ ) results in the condition

$$
\lambda_{4}+\lambda_{5}<0
$$

As in the standard 2HDM, scalars $D_{H}$ and $D_{A}$ have opposite $P$-parities but since they don't couple to fermions, there is no way to assign to them a definite value of $P$-parity. However, their relative parity does matter and for example, vertex $Z D_{H} D_{A}$ is allowed while vertices $Z D_{H} D_{H}$ and $Z D_{A} D_{A}$ are forbidden. Since $\lambda_{5}<0(\mathbb{2} 2$.$) the "scalar" D_{H}$ is lighter than "pseudoscalar".

\subsection{Inert-like vacuum $I_{2},\left\langle\phi_{S}\right\rangle=0$}

The inert-like vacuum $I_{2}$ looks as "mirror-symmetric" to the inert vacuum $I_{1}$. The interactions among scalars and between scalars and gauge bosons in both cases are identical in form with the change $\phi_{S} \leftrightarrow \phi_{D}$. The only difference between $I_{2}$ and $I_{1}$ is given by the Yukawa interaction.

Main formulae for this state are similar to those for the vacuum $I_{1}$ with obvious replacements. The corresponding field decomposition is given by

$$
\phi_{S}=\left(\begin{array}{c}
S^{+} \\
\frac{S_{H}+i S_{A}}{\sqrt{2}}
\end{array}\right), \quad \phi_{D}=\left(\begin{array}{c}
G^{+} \\
\frac{v+h_{D}+i G}{\sqrt{2}}
\end{array}\right),
$$

with one Higgs particle $h_{D}$ and four $S$-particles: $S_{H}, S_{A}, S^{ \pm}$.

Symmetry properties. The inert-like vacuum $I_{2}$ violates $D$-symmetry ([2.5). Moreover, here $S$-parity is also violated by the Yukawa interaction (in contrast to the $D$-parity in the inert vacuum).

Allowed regions of parameters. The inert-like extremum exists only for $m_{22}^{2}>0$. It can be vacuum if $m_{11}^{2} / \sqrt{\lambda_{1}}<m_{22}^{2} / \sqrt{\lambda_{2}}$. For $R^{2}>1$ there are no additional demands. If $R^{2}<1$ inert-like extremum can be a vacuum only if at least one of quantities $v_{S}^{2}, v_{D}^{2}$, defined by eq. (4.7), appears to be negative. Both these conditions are similar to those for the inert vacuum $I_{1}$.

Particle properties. The masses of the Higgs boson $h_{D}$ and $S$-scalars are given by (cf. (5.3]))

$$
\begin{gathered}
M_{h_{D}}^{2}=\lambda_{2} v^{2}=m_{22}^{2}, \quad M_{S^{ \pm}}^{2}=\frac{\lambda_{3} v^{2}-m_{11}^{2}}{2}, \\
M_{S_{A}}^{2}=M_{S^{ \pm}}^{2}+\frac{\lambda_{4}-\lambda_{5}}{2} v^{2}, \quad M_{S_{H}}^{2}=M_{S^{ \pm}}^{2}+\frac{\lambda_{4}+\lambda_{5}}{2} v^{2} .
\end{gathered}
$$

The Higgs boson $h_{D}$ couples to gauge bosons just as the Higgs boson of the SM, however it does not couple to fermions at the tree level. The $S$-scalars do interact with fermions. All fermions, by construction interacting only with $\phi_{S}$ with vanishing v.e.v. $\left\langle\phi_{S}\right\rangle=0$, are massless. (Small mass can appear only as a loop effect.) Here there are no candidates for dark matter particles. 


\subsection{Mixed vacuum $M,\left\langle\phi_{S}\right\rangle,\left\langle\phi_{D}\right\rangle \neq 0$}

The mixed extremum $M$ violates both $D$ - and $S$-symmetries. In this vacuum we have massive fermions and no candidates for DM particle, like in the SM. The decomposition around the mixed vacuum looks as follows:

$$
\phi_{S}=\left(\begin{array}{c}
\rho_{S}^{+} \\
\frac{v_{S}+\rho_{S}+i \chi_{S}}{\sqrt{2}}
\end{array}\right), \phi_{D}=\left(\begin{array}{c}
\rho_{D}^{+} \\
\frac{v_{D}+\rho_{D}+i \chi_{D}}{\sqrt{2}}
\end{array}\right),
$$

where the $\rho_{S}^{+}$and $\rho_{D}^{+}$lead to two orthogonal combinations $G^{+}$and $H^{+}$, while $\rho_{S}$ and $\rho_{D}\left(\chi_{S}\right.$ and $\left.\chi_{D}\right)$ - to two orthogonal combinations $h$ and $H(G$ and $A)$, respectively. There are here five Higgs bosons - two charged $H^{ \pm}$and three neutral ones: the CP-even $h$ and $H$ and CP-odd $A$.

Allowed regions of parameters. In accordance with (4.7) and (4.8) the mixed extremum is global minimum of potential, i. e. vacuum, if and only if $v_{S}^{2}>0, v_{D}^{2}>0$ and $R^{2}<1$. For v.e.v.'s squared given by eqs. (4.7) the latter conditions can be transformed to the relations between mass parameters $m_{11}^{2}$ and $m_{22}^{2}$ :

$$
\begin{aligned}
& \text { at } \quad 1>R>0: \quad 0<R \frac{m_{11}^{2}}{\sqrt{\lambda_{1}}}<\frac{m_{22}^{2}}{\sqrt{\lambda_{2}}}<\frac{m_{11}^{2}}{R \sqrt{\lambda_{1}}} ; \\
& \text { at } 0>R>-1: \frac{m_{22}^{2}}{\sqrt{\lambda_{2}}}>R \frac{m_{11}^{2}}{\sqrt{\lambda_{1}}}, \frac{m_{22}^{2}}{\sqrt{\lambda_{2}}}>\frac{m_{11}^{2}}{R \sqrt{\lambda_{1}}} .
\end{aligned}
$$

Particle properties. Masses of charged and axial scalars together with mass matrix for CPeven scalars $\mathscr{M}$ are (see, e.g. [目, 团])

$$
M_{H^{ \pm}}^{2}=-\frac{\lambda_{4}+\lambda_{5}}{2} v^{2}, \quad M_{A}^{2}=-v^{2} \lambda_{5} \quad\left(v^{2}=v_{S}^{2}+v_{D}^{2}\right), \quad \mathscr{M}=\left(\begin{array}{cc}
\lambda_{1} v_{S}^{2} & \lambda_{345} v_{S} v_{D} \\
\lambda_{345} v_{S} v_{D} & \lambda_{2} v_{D}^{2}
\end{array}\right) .
$$

The masses of the neutral CP-even Higgs bosons are given by eigenvalues of this mass matrix.

The extremum can be minimum only if both diagonal elements of mass matrix and its determinant are positive, i. e. $\lambda_{1} \lambda_{2} v_{S}^{2} v_{D}^{2}\left(1-R^{2}\right)>0$, in agreement with the above mentioned conditions. It means also that in the case if mixed extremum is minimum, it is global minimum - vacuum.

Couplings of the physical Higgs bosons to fermions and gauge bosons have standard forms as for the 2HDM, with the Model I Yukawa interaction.

\section{Evolution of phase states of the Universe}

In this section we consider possible phase history of the Universe, leading to the inert vacuum $I_{1}$ today, based on the thermal evolution described in sec. 3 . To summarize properties of different vacua and to classify all possible ways of evolution of the Universe we will use phase diagrams in the $\left(\mu_{1}(T), \mu_{2}(T)\right)$ plane, where

$$
\mu_{1}(T)=m_{11}^{2}(T) / \sqrt{\lambda_{1}}, \quad \mu_{2}(T)=m_{22}^{2}(T) / \sqrt{\lambda_{2}} .
$$

Let us remind (sect. [3) that in our approximation during cooling down of Universe parameters $\lambda_{i}$ are fixed, while mass parameters $m_{i i}^{2}$ vary. These variations result in modification of vacuum 
state and a possible change of its nature. Possible types of evolution depend on value of parameter $R(\mathbb{2} .3)$ and are depicted in the figures $\square, \square$ and $\mathrm{B}$. The possible current states of Universe are represented in these figures by small black dots $P=\left(\mu_{1}, \mu_{2}\right)$. Since currently we are in the inert phase, we have $\mu_{1}>0$ for each point $P$ (see sect. 52 ). The parameter $\mu_{2}$ can be both positive (points $P 1$ and $P 3$ ) and negative (points $P 2, P 4$ and $P 5$ ).

In accordance with (3.2) a particular evolution leading to a given physical vacuum state $P$ is represented by a ray, that ends at a point $P$. Arrows on these rays are directed towards a growth of time (decreasing of temperature). The direction of the ray is determined by parameters (cf. (B.2.))

$$
\tilde{c}_{1}=c_{1} / \sqrt{\lambda_{1}}, \quad \tilde{c}_{2}=c_{2} / \sqrt{\lambda_{2}}, \quad \tilde{c}=\tilde{c}_{2} / \tilde{c}_{1} .
$$

According to (B.2), for $\tilde{c}>0$ in the initial state of Universe $(T \rightarrow \infty) m_{11}^{2}<0$ and $m_{22}^{2}<0$, i. e. the initial phase state of the Universe is electroweak symmetric. If $\tilde{c}<0$ in the initial state of Universe either $m_{11}^{2}$ or $m_{22}^{2}$ is positive, i. e. the initial state of Universe is

\begin{tabular}{|c|c|c|}
\hline $\mathrm{R}$ & $\tilde{c}$ & initial state of Universe \\
\hline$>0$ & $>0$ & $E W s$ \\
\hline$<0$ & $>0$ & $E W s$ \\
& $<0$ & $E W v$ \\
\hline
\end{tabular}
electroweak-symmetry violating ${ }^{3}-E W v$. Taking into account (B.4) the list of opportunities is presented in the Table here.

For different possible positions of today's point $P$ we consider typical evolutions for different possible values of parameter $\tilde{c}$. In figures below all representative rays are shown; they are labeled by two numbers, with the first one corresponding to the label of the final point $P$.

\subsection{The case $R>1$}

Phase diagram for this case is presented in Fig. 四. It contains one quadrant with $E W s$ phase and two sectors, describing the $I_{1}$ and $I_{2}$ phases. These sectors are separated by the phase transition line $\mu_{1}=\mu_{2}$ (thick black line). Two typical positions of today's state are represented by points $P 1\left(\mu_{2}>0\right)$ and $P 2\left(\mu_{2}<0\right)$. Since (according to (B.4)), both $\tilde{c}_{1}, \tilde{c}_{2}>0(\tilde{c}>0)$, all possible phase evolutions are represented by rays 11 and 12 for the today's point $P 1$ and by a ray 21 which leads to the today's point $P 2$.

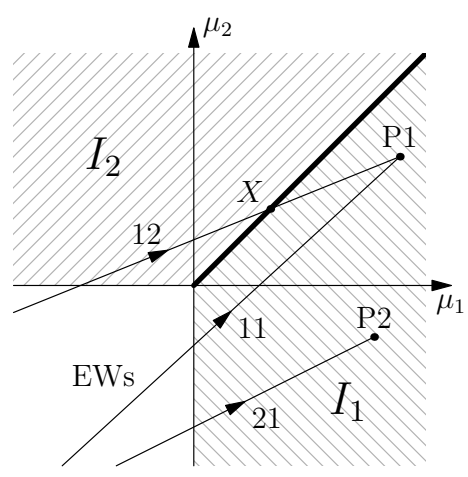

Figure 1: $R>1$ case.

Ray 11: $\tilde{c}>\boldsymbol{\mu}_{2} / \boldsymbol{\mu}_{1}>0$. The Universe started from the EWs state and after the second-order EWSB transition at $m_{11}^{2}(T)=0$, i. e. it has entered to the present inert phase $I_{1}$ at the temperature

$$
T_{E W s, 1}=\sqrt{m_{11}^{2} / c_{1}}=\sqrt{\mu_{1} / \tilde{c}_{1}} .
$$

Ray 12: $0<\tilde{c}<\mu_{2} / \mu_{1}$. The Universe started from the $E W s$ state. Then it went through the EWSB second-order phase transition into the inert-like phase $I_{2}$ at $m_{22}^{2}(T)=0$, i. e. at the temperature

$$
T_{E W s, 2}=\sqrt{m_{22}^{2} / c_{2}}=\sqrt{\mu_{2} / \tilde{c}_{2}}
$$

\footnotetext{
${ }^{2}$ We distinguish present day values of parameters $\mu_{i}$ and their values $\mu_{i}(T)$ at some temperature $T$.

${ }^{3}$ Such opportunity is not ruled out [[0], but it contradicts a key idea of modern approach - the initial state of Universe has high symmetry which is broken at cooling down. In this sense such opportunity is unnatural.
} 
The next transition is the phase transition from the inert-like phase $I_{2}$ into the today's inert phase $I_{1}$ at the point $R$, where $\mu_{2}(T)=\mu_{1}(T)$, i. e. at the temperature

$$
T_{2,1}=\sqrt{\left(\mu_{1}-\mu_{2}\right) /\left(c_{1}-\tilde{c}_{2}\right)} .
$$

That is the first-order phase transition with the latent heat given by

$$
Q_{I_{2} \rightarrow I_{1}}=T_{2,1}\left(\partial \mathscr{E}_{I_{2}} / \partial T-\partial \mathscr{E}_{I_{1}} / \partial T\right)_{T=T_{2,1}}=\left(\mu_{2} \tilde{c}_{1}-\mu_{1} \tilde{c}_{2}\right) T_{2,1}^{2} / 4 .
$$

Ray 21: $\boldsymbol{\mu}_{2}<0$. The Universe started from $E W s$ state and after the second-order EWSB transition at the temperature (6.3) has entered the today's phase.

\subsection{The case $1>R>0$}

The phase diagram for this case - Fig. $\square$ is obtained from that for previous case (Fig. 四) by adding in the upper right quadrant the new sector - the mixed phase $M$, described in accordance with (5.8) by equation

$$
0<R \mu_{1}<\mu_{2}<\mu_{1} / R
$$

As before, since $R>0$ we have $\tilde{c}>0$.

Since currently we are in the inert vacuum, the possible today's states are of type of points $P 3$ and $P 4$, for which

$$
\mu_{2}<R \mu_{1} .
$$

All possible phase evolutions are represented by three rays

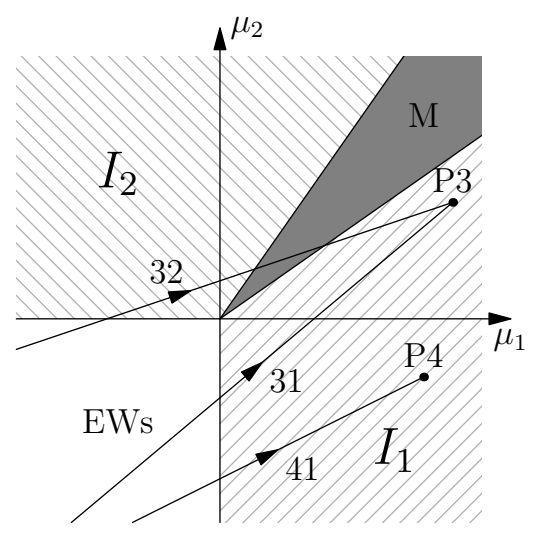

Figure 2: $1>R>0$ case. in Fig. $\square$, with rays 31 and 32 having the today's endpoint $P 3$ while the ray 41 is pointing $P 4$.

For the rays 31 and 41 , phase evolutions are as for the rays 11 and 21, respectively. New situation appears for the ray 32 .

Ray 32: $0<\tilde{c}<\mu_{2} / \boldsymbol{\mu}_{1}$. The Universe started from the $E W s$ state. Then at the temperature given by (6.4) it went through the EWSB second-order phase transition into the inert-like phase $I_{2}$. At the subsequent cooling down the Universe goes through the mixed phase $M$ into the present inert phase $I_{1}$. The second-order phase transitions $I_{2} \rightarrow M$ and $M \rightarrow I_{1}$ happened at the temperatures

$$
\begin{array}{ll}
T_{p h t r}: & T_{2, M}=\sqrt{\left(\mu_{1}-R \mu_{2}\right) /\left(\tilde{c}_{1}-R \tilde{c}_{2}\right)} \\
T_{M, 1}=\sqrt{\left(R \mu_{1}-\mu_{2}\right) /\left(R \tilde{c}_{1}-\tilde{c}_{2}\right)}
\end{array}
$$

In accordance with equations in sect. [, at the transition point $I_{2} \rightarrow M$ masses of $S_{H}$ and $h$ vanish, while at the transition point $M \rightarrow I_{1}$ masses of $h$ and $D_{H}$ become 0 . At small distance from the transition point with temperature $T_{p h t r}$ these masses grow as a function of the temperature $T$ as $M_{a}^{2}=A_{a}\left|T^{2}-T_{p h t r}^{2}\right|$, with different coefficients $A_{a}$.

\subsection{The case $0>R>-1$}

The phase diagram is presented in Fig. [3]. In this case, as follows from (5.8), the mixed phase $M$ region is realized in a wider region than in Fig. \, even beyond an upper right quadrant of this plane, namely:

$$
\mu_{2}>\mu_{1} / R, \quad \mu_{2}>\mu_{1} R .
$$


Since currently we are in the inert vacuum $\left(\mu_{1}>0\right)$, for the today's point $P 5$ we have $\mu_{2}<R \mu_{1}\left(\mu_{2}<0\right)$. New opportunities appear due to larger freedom for temperature coefficients $c_{i}$, as in accordance with (B.4) in this case $\tilde{c}$ can be negative.

All possible phase evolutions leading to the point $P 5$ are represented in Fig. [1] by four rays 51, 52, 53 and 54.

The ray 51 with $\tilde{c}>0$ describes similar evolution as rays 21 and 41 . New are rays 52, 53 and 54 (at $\tilde{c}<0$ ) with common feature, which is a lack of electroweak symmetry in very early stages of the Universe.

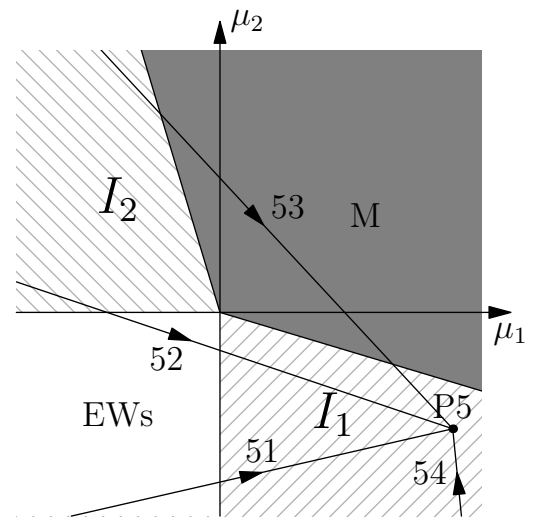

Figure 3: $1>R>0$ case.

Ray 52: $\tilde{\boldsymbol{c}}_{\mathbf{1}}>\mathbf{0}, \tilde{\boldsymbol{c}}_{\mathbf{2}}<\mathbf{0}, \tilde{\boldsymbol{c}}>\boldsymbol{\mu}_{2} / \boldsymbol{\mu}_{\mathbf{1}}$. Here a high-temperature state of the Universe is the inert-like vacuum $I_{2}$. With cooling down the Universe goes through electroweak symmetric phase $E W s$ into the present $I_{1}$ phase. The second-order phase transitions $I_{2} \rightarrow E W s$ and $E W s \rightarrow I_{1}$ happened, respectively, at the temperatures

$$
T_{2, E W s}=\sqrt{\mu_{2} / \tilde{c}_{2}}, \quad T_{E W s, 1}=\sqrt{\mu_{1} / \tilde{c}_{1}} .
$$

Ray 53: $\tilde{\boldsymbol{c}}_{\mathbf{1}}>\mathbf{0}, \tilde{\boldsymbol{c}}_{\mathbf{2}}<0, \tilde{\boldsymbol{c}}<\boldsymbol{\mu}_{2} / \boldsymbol{\mu}_{\mathbf{1}}$. Here a high-temperature state of the Universe is an inert-like vacuum $I_{2}$. With cooling down the Universe passes through the mixed phase $M$ into the present $I_{1}$ phase. The phase transitions $I_{2} \rightarrow M$ and $M \rightarrow I_{1}$ are of the second order; they happened at the temperatures given by eqs. (6.9).

Ray 54: $\tilde{c}_{\mathbf{1}}<\mathbf{0}, \tilde{c}_{\mathbf{2}}>\mathbf{0}$. For this ray the Universe stays in the inert vacuum $I_{1}$ during the whole evolution.

\section{Results and discussion}

Main results. The most important observation we made in this paper is as follows: If current state of the Universe is described by IDM, then during the thermal evolution the Universe can pass through various intermediate phases, different from the inert one. These possible intermediate phases contain no dark matter, which appears only at the relatively late stage of cooling down of the Universe.

We find that in the considered approximation the thermal evolution of Universe can be studied effectively in the $\left(\mu_{1}, \mu_{2}\right)$ plane, at fixed values of quartic parameters $\lambda_{i}$. Different types of such evolution represented as directed rays depend crucially on two parameters: $R([2.3)$, describing the allowed for various vacua regions of the $\left(\mu_{1}, \mu_{2}\right)$ plane, and $\tilde{c}$ ( 6.2$)$, determining the direction of rays. The first one depends only on the ratios between coefficients of quartic part of potential $\lambda_{i}$, while the second depends both on mentioned parameters $\lambda_{i}$ and on the known gauge and Yukawa couplings.

The starting point of evolution of Universe to the present day inert phase state can be either electroweak symmetric (EWs) state (if $\tilde{c}>0$ ) or electroweak symmetry violating state (at $\tilde{c}<0$ ). A complete set of possible ways of evolution of the Universe can be summarized as follows (symbol 
I or II over arrows corresponds to the type of phase transition):

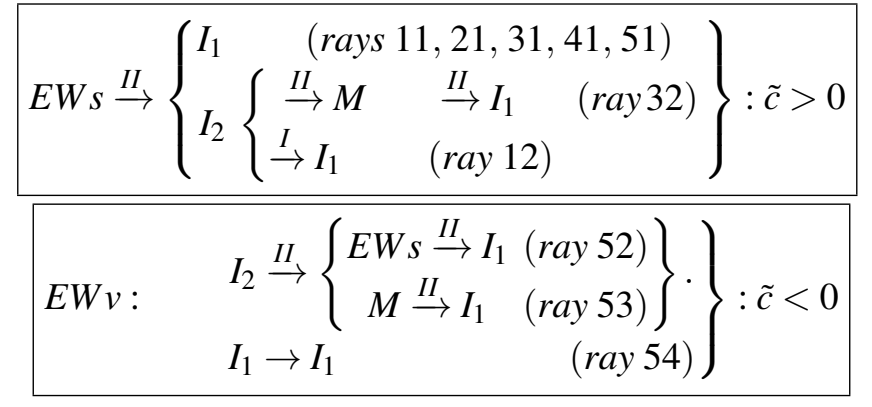

To find what scenario of evolution is realized in nature, one should measure all parameters of potential. The program how to measure these parameters at LHC and ILC is under preparation.

\section{Outlook.}

A. Extra phase transitions at lower temperature than EWSB temperature (and especially first order phase transition at the evolution like ray 12) can influence for baryogenesis even stronger than transformation of standard second order EWSB transition into the first order one due to term $\phi^{3} T[\mathbb{[}]$. Moreover, in contrast to the standard picture, the considered scenarios allow for the phase transition to the current inert phase at relatively low temperature, giving new starting point for calculation of a today's abundance of the neutral DM components of the Universe and other phenomena.

B. In this paper we calculated thermal evolution of the Universe in the very high temperature approximation, i. e. for $T^{2} \gg\left|m_{i i}^{2}\right|$. The most interesting effects are expected at lower temperatures, where more precise calculations are necessary. The simplest expected modifications of the presented description are:

1. Appearance of cubic terms like $\phi^{3} T[\mathbb{W}]$. These terms are important near phase transition point, as they can transform some second-order phase transition into the first-order transition.

2. The parameters become depend on temperature in more complicated way than that given by (B.2). Therefore, the rays, depicted thermal evolutions in Figs. $\square, \square$ and $\mathbf{Z}$, can become non-straight. The bending of these rays can be different in different points of our plots and at different $\lambda_{i}$. It can give possible spectrum of phase evolutions even reacher that discussed above.

However, we expect that the general picture will not change too much.

\section{References}

[1] N. G. Deshpande and E. Ma, Phys. Rev. D 18 (1978) 2574; R. Barbieri, L. J. Hall and V. S. Rychkov, Phys. Rev. D 74 (2006) 015007 [arXiv:hep-ph/0603188].

[2] I. F. Ginzburg, K.A. Kanishev, M. Krawczyk and D. Sokołowska. hep-ph/1009.4953

[3] Q. H. Cao, E. Ma and G. Rajasekaran, Phys. Rev. D 76 (2007) 095011 [arXiv:0708.2939 [hep-ph]]; E. Dolle, X. Miao, S. Su and B. Thomas, Phys. Rev. D 81, 035003 (2010) [arXiv:0909.3094 [hep-ph]]; C. Arina, F. S. Ling and M. H. G. Tytgat, JCAP 0910 (2009) 018 [arXiv:0907.0430 [hep-ph]]; T. Hambye and M. H. G. Tytgat, Phys. Lett. B 659 (2008) 651 [arXiv:0707.0633 [hep-ph]]; E. Nezri, M. H. G. Tytgat and G. Vertongen, JCAP 0904 (2009) 014 [arXiv:0901.2556 [hep-ph]]; S. Andreas, 
M. H. G. Tytgat and Q. Swillens, JCAP 0904 (2009) 004 [arXiv:0901.1750 [hep-ph]]; S. Andreas, T. Hambye and M. H. G. Tytgat, JCAP 0810 (2008) 034 [arXiv:0808.0255 [hep-ph]]; L. Lopez Honorez, E. Nezri, J. F. Oliver and M. H. G. Tytgat, JCAP 0702 (2007) 028 [arXiv:hep-ph/0612275]; L. L. Honorez and C. E. Yaguna, arXiv:1003.3125 [hep-ph]; arXiv:1011.1411 [hep-ph]; M. Gustafsson, E. Lundstrom, L. Bergstrom and J. Edsjo, Phys. Rev. Lett. 99 (2007) 041301 [arXiv:astro-ph/0703512]; E. Lundstrom, M. Gustafsson and J. Edsjo, Phys. Rev. D 79 (2009) 035013 [arXiv:0810.3924 [hep-ph]]. M. Krawczyk and D. Sokołowska, arXiv:0911.2457 [hep-ph].

[4] I. F. Ginzburg and K. A. Kanishev, Phys. Rev. D 76 (2007) 095013 [arXiv:0704.3664 [hep-ph]].

[5] I.F. Ginzburg Acta Phys. Polonica B 37 (2006) 1161-1172, hep-ph / 0512102 ; I. F. Ginzburg, I. P. Ivanov and K. A. Kanishev, Phys. Rev. D 81 (2010) 085031 [arXiv:0911.2383 [hep-ph]].

[6] I. F. Ginzburg and M. Krawczyk, Phys. Rev. D 72 (2005) 115013 [arXiv:hep-ph/0408011]. D. Sokołowska, University of Warsaw, Master Thesis 2007

[7] J. Velhinho, R. Santos and A. Barroso, Phys. Lett. B 322, 213 (1994); S. Nie and M. Sher, Phys. Lett. B 449, 89 (1999) [arXiv:hep-ph/9811234]; S. Kanemura, T. Kasai and Y. Okada, Phys. Lett. B 471 (1999) 182 [arXiv:hep-ph/9903289]; B. M. Kastening, arXiv:hep-ph/9307224.

[8] I. P. Ivanov, Acta Phys. Polon. B 40 (2009) 2789 [arXiv:0812.4984 [hep-ph]].

[9] J. L. Diaz-Cruz and A. Mendez, Nucl. Phys. B 380 (1992) 39. P. M. Ferreira, R. Santos and A. Barroso, Phys. Lett. B 603, 219 (2004) [Erratum-ibid. B 629, 114 (2005)] [arXiv:hep-ph/0406231]; A. Barroso, P. M. Ferreira and R. Santos, Phys. Lett. B 632, 684 (2006) [arXiv:hep-ph/0507224]. A. Barroso, P. M. Ferreira and R. Santos, Phys. Lett. B 652, 181 (2007) [arXiv:hep-ph/0702098]; A. Barroso, P. M. Ferreira, R. Santos and J. P. Silva, Phys. Rev. D 74 (2006) 085016 [arXiv:hep-ph/0608282].

[10] S. Weinberg, Phys. Rev. D 9 (1974) 3357; L. Dolan and R. Jackiw, Phys. Rev. D 9, 3320 (1974); Y. Fujimoto and S. Sakakibara, Phys. Lett. B 151 (1985) 260; M. B. Gavela, O. Pene, N. Rius and S. Vargas-Castrillon, Phys. Rev. D 59 (1999) 025008 [arXiv:hep-ph/9801244]; G. R. Dvali and K. Tamvakis, Phys. Lett. B 378 (1996) 141 [arXiv:hep-ph/9602336]; R. N. Mohapatra and G. Senjanovic, Phys. Lett. B 89 (1979) 57, Phys. Rev. D 20 (1979) 3390, Phys. Rev. Lett. 42 (1979) 1651; B. Bajc, arXiv:hep-ph/0002187.

[11] N. Turok and J. Zadrozny, Nucl. Phys. B 369 (1992) 729. A. I. Bochkarev, S. V. Kuzmin and M. E. Shaposhnikov, Phys. Lett. B 244 (1990) 275. V. Jain and A. Papadopoulos, Phys. Lett. B 314, 95 (1993) [arXiv:hep-ph/9303282]; S. Kanemura, Y. Okada and E. Senaha, Phys. Lett. B 606, 361 (2005) [arXiv:hep-ph/0411354] L. Fromme, S. J. Huber and M. Seniuch, JHEP 0611, 038 (2006) [arXiv:hep-ph/0605242]. 